\title{
Spatio-Temporal Inertial Measurements Feature Extraction Improves Hand Movement Pattern Recognition without Electromyography
}

\author{
Rami N. Khushaba ${ }^{1}$, Agamemnon Krasoulis ${ }^{2}$, Adel Al-Jumaily ${ }^{1}$, and Kianoush Nazarpour ${ }^{2,3}$
}

\begin{abstract}
Recent studies indicate the limited clinical acceptance of myoelectric prostheses, as upper extremity amputees need improved functionality and more intuitive, effective, and coordinated control of their artificial limbs. Rather than exclusively classifying the electromyogram (EMG) signals, it has been shown that inertial measurements (IMs) can form an excellent complementary signal to the EMG signals to improve the prosthetic control robustness. We present an investigation into the possibility of replacing, rather than complementing, the EMG signals with IMs. We hypothesize that the enhancements achieved by the combined use of the EMG and IM signals may not be significantly different from that achieved by the use of Magnetometer (MAG) or Accelerometer (ACC) signals only, when the temporal and spatial information aspects are considered. A large dataset comprising recordings with 20 ablebodied and two amputee participants, executing 40 movements, was collected. A systematic performance comparison across a number of feature extraction methods was carried out to test our hypothesis. Results suggest that, individually, each of the ACC and MMG signals can form an excellent and potentially independent source of control signal for upper-limb prostheses, with an average classification accuracy of $\approx 93 \%$ across all subjects. This study suggests the feasibility of moving from surface EMG to IM signals as a main source for upper-limb prosthetic control in real-life applications.
\end{abstract}

\section{INTRODUCTION}

Traditional myoelectric control utilizes the surface electromyogram (EMG) signals from the stump remnant muscles to extract the prosthesis control information [1]. In academic research, EMG pattern recognition has been very successful in decoding grip type, wrist and individual and combined fingers movements [2]-[4]. Despite the potential offered by myoelectric hands, prosthesis users report these devices to be challenging to control and limited in function [5], [6]. This limitation has been mainly attributed to the lack of classification robustness and a simultaneous requirement for a large number of EMG electrodes [7]. Hence, many research

\footnotetext{
*A. Krasoulis was supported by grants EP/F500385/1 and BB/F529254/1 for the University of Edinburgh School of Informatics Doctoral Training Centre in Neuroinformatics and Computational Neuroscience (www.anc.ac.uk/dtc) from the UK Engineering and Physical Sciences Research Council (EPSRC), UK Biotechnology and Biological Sciences Research Council (BBSRC), and the UK Medical Research Council (MRC). The work of K. Nazarpour is supported by UK Engineering and Physical Sciences Research Council (EP/R004242/1).

1 Faculty of Engineering and Information Technology, University of Technology, Sydney (UTS), 15 Broadway Ultimo, Australia where Rami N. Khushaba is a visiting fellow.

2 School of Engineering, Newcastle University, Newcastle-upon-Tyne NE1 7RU, UK.

3 Institute of Neuroscience, Newcastle University, Newcastle-upon-Tyne NE2 4HH, UK.

Emails: rami.khushaba@uts.edu.au, agamemnon.krasoulis@gmail.com, Adel.Al-Jumaily@uts.edu.au, kianoush.nazarpour@newcastle.ac.uk
}

groups have moved towards multi-modal control solutions by considering inertial measurements (IMs) as a complementary source signal to EMG [7], [8].

Scheme et al. [9] and Fougner et al. [10] were among the first to consider the combination of EMG time-domain (TD) features with the average value of accelerometer (ACC) readings for prosthesis control. Boschmann et al. [11] demonstrated that a classifier trained with features from EMG, ACC, and gyroscope (GYRO) outperforms classifiers trained using only EMG or EMG + ACC data when classifying transient EMG data. Simple TD features combined with AutoRegressive (AR) model parameters (a combination referred to as TDAR) were extracted from the EMG signals, while the mean average value plus wavelength were considered for ACC feature extraction and mean average value plus root mean square were considered for GYRO. Geng et al. [12] considered the combination of the EMG and ACCmechanomyography signals and performed their analysis on amputees and intact-limbed subjects. Similar to previous studies, simple TD features were considered for ACCmechanomyography signals: mean absolute value, variance, and maximum value. The combination of EMG+ACC was also considered by Radmand et al. [13] using TD features for the EMG data and the mean value of the ACC data. Krasoulis et al. [7] considered the mean value of the combination of ACC, GYRO, and Magnetomyogram (MAG) signals in concatenation with the TD features from the EMG signals to show significant enhancements in classification accuracies in a problem with 40 classes of movements. Khushaba et al. [14] as well used the mean value of ACC signals and showed reduction in classification error.

The literature has always considered other sensing modalities such as ACC, GYRO, and MAG as supporting signals to the EMG and not as competing source of information. This could be due to the fact that previous studies were focused on extracting IM features that capture the temporal content, without further investigation into the spatial content that may reveal how different channels interact. Our hypothesis in this study is that movement classification can be enhanced if spatio-temporal information of IMs is considered. This hypothesis is motivated by recent studies which show significant performance improvement when both temporal and spatial information are extracted from EMG [15], [16].

\section{METHODS}

\section{A. Graph Laplacian-based Feature Extraction}

A graph is a structure involving a set of objects with each pair of objects being in some sense related [17]. Given 
a graph $\mathcal{G}=(\mathcal{V}, \mathcal{E})$ with $m$ vertices, each vertex in $\mathcal{V}$ represents a channel (ACC or MAG), and $\mathcal{E}$ is a set of edges connecting spatially distributed channels. Let $\mathbf{A}$ be a symmetric $m \times m$ adjacency matrix with its entries $\mathbf{A}_{i j}$ having the weight of the edge joining channel $i$ and channel $j$. Under these definitions, the Laplacian matrix $\mathbf{L}_{m \times m}$ of a graph $\mathcal{G}$ with $m$ vertices is defined as $\mathbf{L}=\mathbf{D}-\mathbf{A}$ where $\mathbf{D}=\operatorname{diag}(\operatorname{sum}(\mathbf{A}))$ is a diagonal matrix having the degrees along its diagonal, i.e., it contains information about the number of edges attached to each vertex [17]. The smaller the value of the diagonal entries of $\mathbf{D}$, denoted as $\mathbf{D}_{i i}$ (corresponding to channel $i$ ), the more "similar" is channel $i$ to the remaining channels.

When the graph Laplacian under investigation describes hand movement data with electrodes distributed on the forearm, it is not only the temporal information of the IM signals from each channel that influences the structure of the graph, but also the spatial relationships between the information from different channels. To handle both temporal and spatial information, the weights of the adjacency matrix connecting every pair of nodes $i$ and $j$ are represented by the channels' temporal information $\mathbf{x}_{i}^{f}$ and $\mathbf{x}_{j}^{f}$ and their spatial location $\mathbf{x}_{i}^{p}$ and $\mathbf{x}_{j}^{p}$. A modified adjacency matrix encoding the spatial similarity is then utilized [18]:

$$
\mathbf{A}_{i j}=\left\{\begin{array}{ll}
\frac{\left\|\mathbf{x}_{i}^{f}-\mathbf{x}_{j}^{f}\right\|^{2}}{\sigma_{f}^{2}}+\alpha \frac{\left\|\mathbf{x}_{i}^{p}-\mathbf{x}_{j}^{p}\right\|^{2}}{\sigma_{p}^{2}} & \text { if } i \neq j \\
0 & \text { otherwise }
\end{array},\right.
$$

where $\alpha$ is a weight factor to balance the importance of the spatial component, and $\sigma$ is a normalization factor selected as the maximum value from the corresponding vectors. On the other hand, the indices of the different channels are used for the spatial location component. The (symmetric) normalized Laplacian is then formed as $\mathbf{L}^{\text {sym }}:=\mathbf{D}^{-1 / 2} \mathbf{L D}^{-1 / 2}=\mathbf{I}-$ $\mathbf{D}^{-1 / 2} \mathbf{A} \mathbf{D}^{-1 / 2}$. Since the degree matrix $\mathbf{D}$ is diagonal and positive, its reciprocal square root $\mathbf{D}^{-1 / 2} \mathbf{D}^{-1 / 2}$ is just the diagonal matrix whose diagonal entries are the reciprocals of the positive square roots of the diagonal entries of $\mathbf{D}$. Equivalently, the normalized Laplacian can be written as

$$
\mathbf{L}_{i j}^{\text {sym }}=\left\{\begin{array}{ll}
1 & \text { if } i=j \text { and } \mathbf{D}_{i i} \neq 0 \\
-\frac{\mathbf{A}_{i j}}{\sqrt{\mathbf{D}_{i i} \mathbf{D}_{j j}}} & \text { otherwise }
\end{array} .\right.
$$

The first set of features utilized in this paper is the upper (or lower, since $\mathbf{L}$ is a symmetric matrix) triangular portion of the Laplacian matrix $\mathbf{L}$, that is, $-\frac{\mathbf{A}_{i j}}{\sqrt{\mathbf{D}_{i i} \mathbf{D}_{j j}}}$ given $j>i$ (or similarly $i>j$ ). This feature translates to the integral value of the normalized Euclidean distance between the windowed IM data from every two channels. Our extracted features not only include the temporal information but also the spatial information about the interaction between the data from the different channels. On the other hand, since $\mathbf{D}_{i i}$ is the sum of the similarities of $\mathbf{x}_{i}$ to its nearest neighbors in the $n$ dimensional space, the smaller the $\mathbf{D}_{i i}$ value of a point $\mathbf{x}_{i}$, the more likely it is to be near the center of the cluster containing it. This leads us to take advantage of the fact

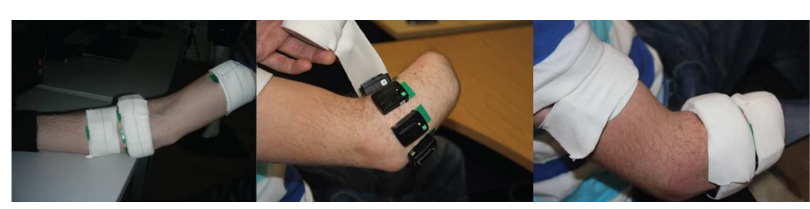

Fig. 1. Sensor placement. Eight EMG-IM sensors were equally spaced around the participants forearm, two targeted the EDC and FDS muscles, and two were placed on the biceps and triceps muscles. Elastic bandage was used to keep sensor positions fixed. Sensor placement shown for an able-bodied (left) and an amputee subject (center, right).

that the points $\mathbf{x}_{i}$ 's that are near their cluster centers should contribute more in variance calculation compared to the $\mathbf{x}_{i}$ 's which are outliers or noise. Hence, to capture the variance information we have included in our extracted features the weighted variance of the graph Laplacian; previously defined as $\sum_{k} x_{i k}^{2} \mathbf{D}_{i i}$ based on the spectral graph theory [16]. In this setting, $k$ is the time sample index with $k=1,2,3, \cdots, N$, and $N$ is the temporal window size.

\section{B. Data Collection}

Twenty intact-limbed subjects and two transradial amputees took part in the experiment as described in [7]. Briefly, data were collected by using a Delsys Trigno ${ }^{\mathrm{TM}}$ IM Wireless System. Each electrode incorporated one EMG sensor and one 9-degree-of-freedom (DOF) IM unit. The IM unit comprised a tri-axial accelerometer, gyroscope, and magnetometer. Therefore, ten raw signals were associated with each EMG-IM sensor. The sampling frequency was set to $2 \mathrm{kHz}$ for EMG and $128 \mathrm{~Hz}$ for IM data. No IM calibration was performed since IM recordings were used in their raw format. Eight sensors were equally spaced around the forearm and were placed $3 \mathrm{~cm}$ below the elbow. Two sensors targeted the extrinsic hand muscles: extensor digitorum communis (EDC) and flexor digitorum superficialis (FDS), and the remaining two were placed on the biceps and triceps brachii muscles. Figure $1^{1}$ shows the electrode placement for one able-bodied subject and one amputee. Upon skin cleansing and electrode placement, participants reproduced a series of 40 motions, including various individuated-finger, hand, wrist, grasping and functional movements. Each movement was repeated six times and trials were interleaved with $5 \mathrm{~s}$ resting periods. The two amputee volunteers performed bilateral mirrored movements. Further details can be found in [7].

\section{Signal preprocessing and classifier training}

A sliding window approach was utilized to extract features from all of the EMG, ACC, and MAG signals, with a window size of $150 \mathrm{~ms}$ shifted by $50 \mathrm{~ms}$. The EMG and IM signals were synchronized with linear interpolation. The number of raw signals (denoted as Nignals) associated with each MAG and ACC sensors was 3 signals / sensor. We used the proposed feature extraction based on the upper triangular portion of adjacency matrix to end up with ( $N$ signals $\times$

\footnotetext{
${ }^{1}$ Figure 1 was originally published in [7] and is licensed under a Creative Commons Attribution 4.0 International License (https://creativecommons.org/licenses/by/4.0/).
} 
$(N$ signals - 1) / 2) features with an additional Nsignals features extracted from the graph variance as indicated in the previous section. Since the dimensionality of the extracted feature set was large, we used the Spectral Regression (SR) dimensionality reduction method [19] to project the total number of features into $c-1$ features, where $c$ is the number of classes. For movement intent decoding from the EMG and IM data, we used a linear discriminant analysis (LDA) classifier.

A leave-one-trial out scheme was utilized to test the proposed IM features, in which we left one complete trial from each movement for extracting the testing feature set while the remaining five trials were used to extract the training feature set. We then averaged the results across all six repetitions. To compare the performance of the proposed graph Laplacian-based feature extraction method, denoted here as GL method, we also included a number of feature extraction methods from the literature, including: the root mean square (RMS), combination of mean absolute value (MAV) and waveform length (WL), the empirical cumulative distribution (ECDF) feature proposed specifically for the ACC data in [20], wavelet features (Symmlet family of wavelets with 5 levels of decomposition), and Hjorth and Barlow features from the BioSig toolbox ${ }^{2}$.

\section{EXPERIMENTAL RESULTS}

The average classification error rates across the intactlimbed, amputees, and the combined set of all subjects are shown in Fig. 2. The average classification error rates with the different feature extraction methods were lower when using the MAG sensor than that achieved with the ACC sensor, except when using the proposed GL based features when both ACC and MAG achieved similar error rates in recognizing the 40 classes of hand movements. The justification for the enhanced performance is related to the fact that all methods, except the proposed GL, only consider the information extracted from the individual channels without relating that to the information carried out by the other channels. In this case, the proposed GL-based feature extraction method achieved, on average, a classification error rate of $5.94 \%$ and $13.54 \%$ across the intact-limbed and amputees using the ACC data while achieving an average of $6.15 \%$ and $10.18 \%$ across the intact-limbed and amputees using the MAG data (Bonferroni-corrected $t$-test, $p=0.75$ ).

Moreover, we considered the classification error rates of using the EMG only with different feature extraction methods, including:

1) TD1 feature set which is made of RMS, integralabsolute-value (IAV), number of zero-crossings (ZC), WL feature, slope-sign change (SSC), and the AR model parameters $\left(5^{t h}\right.$ order);

2) AR-RMS feature set: a combination of RMS and AR model parameters $\left(5^{t h}\right.$ order);

3) TD2 feature set [7]: a combination of MAV, WL, AR $\left(4^{\text {th }}\right.$ order), and the log-variance (LogVar) feature;

${ }^{2}$ Available online in http://biosig.sourceforge.net/index.html.
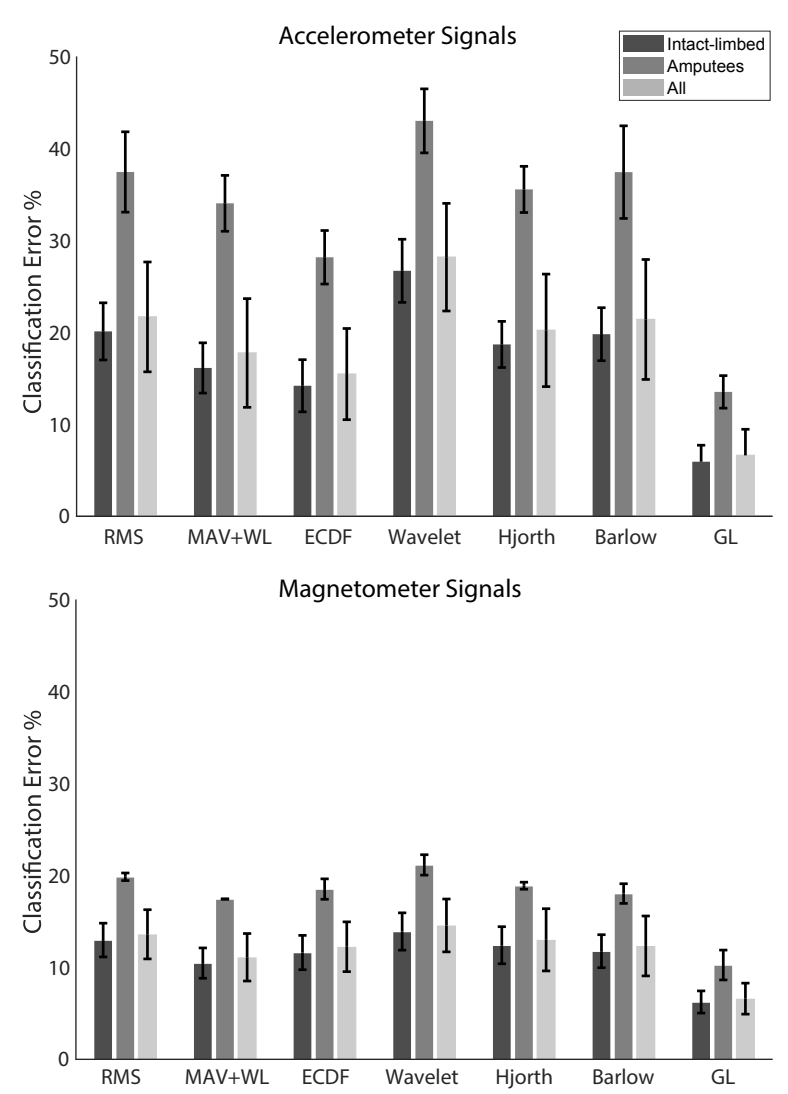

Fig. 2. Average classification errors across the intact-limbed, amputees, and the full set of subjects using different sensors (error bars represent standard deviation).

4) TSD feature set [15]: A combination of temporalspatial time-domain features.

The classification error rate using the EMG signals only are shown in Fig. 3. These results clearly indicate that the error rates achieved by the IM signals with our GL method were significantly lower than that achieved with all feature extraction methods on the EMG data.

Since the ACC and MAG error rates were not significantly different from each other, we focused our attention to the MAG signals. We tested the combination of the MAG (GL features) with EMG (TSD features) signals which achieved an average error of $5.92 \%$ on all subjects in comparison to that of MAG only (achieving average error of $6.52 \%$ across all subjects). In this case, the error rates achieved by the combination of EMG (TSD features) with MAG (GL features) were not significantly different from that of using the MAG features only (Bonferroni-corrected $t$-test, $p=0.25)$. This in turn provides an evidence to support our null hypothesis in that, in the context of our experiments with 20 intact-limbed and 2 transradial amputees, there are no significant enhancements added by the combined EMG+MAG on top of the MAG error rates when both the temporal and the spatial MAG features are considered. 


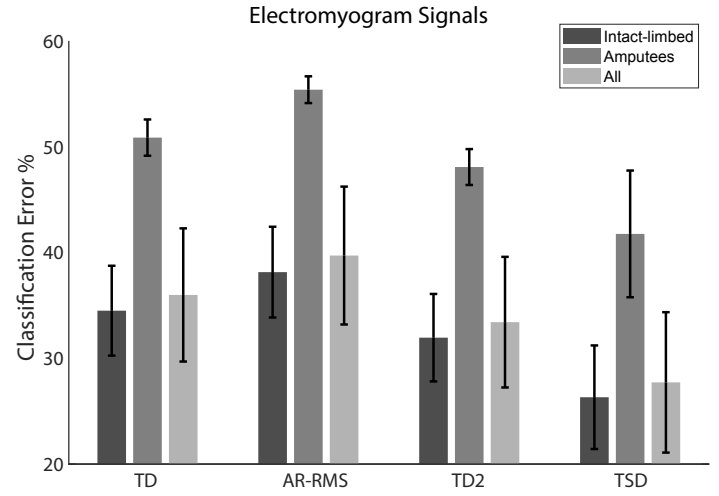

Fig. 3. EMG classification error rates across all subjects with different feature extraction methods (error bars represent standard deviation).

\section{CONCLUSIONS}

We investigated the suitability of replacing the EMG signals with IM signals rather than combining the two, as it is usually done in the myoelectric control literature [7], [10], because of evidence that the latter method results in increased performance when temporal IM information is used. Our experimental results support the hypothesis that if the IM signals features were extracted in a way that considers both the temporal and spatial information aspects, rather than temporal only, then we could significantly reduce classification error, without having to combine IMs with the EMG signals. The finding in this paper is of significant importance as it first indicates the importance of the temporalspatial information extraction and then it reduces the total number of sensors required to operate a prosthetic hand, while avoiding all the well-known non-stationarity problems with the EMG signals. Our findings also agree with the recent literature on the importance of magnetic fields from skeletal muscles as a valuable physiological measurement [21]. We have only tested the proposed IM features on twenty intact-limbed and two transradial amputees offline. Further real-time testing, with a larger group of prosthesis users, is required to determine the efficacy of inertial measurements in prosthesis control.

\section{REFERENCES}

[1] K. Nazarpour, C. Cipriani, D. Farina and T. D. Kuiken, "Advances in control of multi-functional powered upper-limb prostheses," IEEE Transactions on Neural Systems and Rehabilitation Engineering, vol. 22, no. 4, pp. 711-715, 2014.

[2] E. J. Scheme and K. Englehart, "Electromyogram pattern recognition for control of powered upper-limb prostheses: State of the art and challenges for clinical use," Journal of Rehabilitation Research \& Development, vol. 48, no. 6, pp. 643-660, 2011.

[3] G. Li, A. E. Schultz, and T. A. Kuiken, "Quantifying pattern recognitionbased myoelectric control of multifunctional transradial prostheses," IEEE Transactions on Neural Systems and Rehabilitation Engineering. vol. 18, no. 2, pp. 185-192, 2010.

[4] M. Ortiz-Catalan, B. Hkansson and R. Brånemark, "Real-time and simultaneous control of artificial limbs based on pattern recognition algorithms," IEEE Transactions on Neural Systems and Rehabilitation Engineering, vol. 22, no. 4, pp. 756-764, July 2014.
[5] S. M. Engdahl, B. P. Christie, B. Kelly, A. Davis, C. A. Chestek, and D. H. Gates, "Surveying the interest of individuals with upper limb loss in novel prosthetic control techniques," Journal of NeuroEngineering and Rehabilitation. vol. 12, no. 1, p. 53, 2015.

[6] A. Chadwell, L. Kenny, S. Thies, A. Galpin, and J. Head, "The reality of myoelectric prostheses: Understanding what makes these devices difficult for some users to control," Frontiers in Neurorobotics, vol. 10, p. 7, 2016.

[7] A. Krasoulis, I. Kyranou, M. S. Erden, K. Nazarpour, and S. Vijayakumar, "Improved prosthetic hand control with concurrent use of myoelectric and inertial measurements," Journal of NeuroEngineering and Rehabilitation, vol. 14, p. 71, 2017.

[8] D. A. Bennett and M. Goldfarb, "IMU-based wrist rotation control of a transradial myoelectric prosthesis," IEEE Transactions on Neural Systems and Rehabilitation Engineering, vol. PP, no. 99, pp. 1-1., 2017. DOI: $10.1109 /$ TNSRE.2017.2682642

[9] E. Scheme, A. Fougner, Ø. Stavdahl, A. D. C. Chan and K. Englehart, "Examining the adverse effects of limb position on pattern recognition based myoelectric control," 2010 Annual International Conference of the IEEE Engineering in Medicine and Biology, Buenos Aires, pp. 6337-6340, 2010.

[10] A. Fougner, E. Scheme, A. D. C. Chan, K. Englehart and Ø. Stavdahl, "Resolving the limb position effect in myoelectric pattern recognition," IEEE Transactions on Neural Systems and Rehabilitation Engineering, vol. 19 , no. 6, pp. 644-651, 2011.

[11] A. Boschmann, B. Nofen and M. Platzner, "Improving transient state myoelectric signal recognition in hand movement classification using gyroscopes," 35th Annual International Conference of the IEEE Engineering in Medicine and Biology Society (EMBC), Osaka, pp. 6035-6038, 2013.

[12] Y. Geng, P. Zhou, and G. Li, "Toward attenuating the impact of arm positions on electromyography pattern-recognition based motion classification in transradial amputees," Journal of NeuroEngineering and Rehabilitation, vol. 9, no. 1, p. 74, 2012.

[13] A. Radmand, E. Scheme, and K. Englehart, "On the suitability of integrating accelerometry data with electromyography signals for resolving the effect of changes in limb position during dynamic limb movement," Journal of Prosthetics and Orthotics, vol. 26, no. 4, pp. 185-193, 2014.

[14] R. N. Khushaba, A. Al-Timemy, S. Kodagoda and K. Nazarpour, "Combined influence of forearm orientation and muscular contraction on EMG pattern recognition," Expert Systems with Applications, vol. 61, pp. 154-161, 2016.

[15] R. N. Khushaba, A. H. Al-Timemy, A. Al-Ani and A. Al-Jumaily, "A framework of temporal-spatial descriptors-based feature extraction for improved myoelectric pattern recognition," IEEE Transactions on Neural Systems and Rehabilitation Engineering, vol. 25, no. 10, pp. 1821-1831, 2017.

[16] A. Ebied, L. Spyrou, E. Kinney-Lang and J. Escudero, "On the use of higher-order tensors to model muscle synergies," 39th Annual International Conference of the IEEE Engineering in Medicine and Biology Society (EMBC), Seogwipo, pp. 1792-1795, 2017.

[17] F. R. K. Chung, "Spectral graph theory", Regional Conference Series in Mathematics, no. 92, 1997.

[18] N. D. Cahill, W. Czaja, and D. W. Messinger, "Schroedinger Eigenmaps with nondiagonal potentials for spatial-spectral clustering of hyperspectral imagery", Proceedings of Society of Photo-Optical Instrumentation Engineers (SPIE), vol. 9088, pp. 1-13, 2014.

[19] D. Cai, X. He and J. Han, "SRDA: An efficient algorithm for largescale discriminant analysis", IEEE Transactions on Knowledge and Data Engineering, vol. 20, no. 1, pp. 1-12, 2008.

[20] N. Y. Hammerla, R. Kirkham, P. Andras, T. Ploetz, "On preserving statistical characteristics of accelerometry data using their empirical cumulative distribution", Proceedings of the 2013 International Symposium on Wearable Computers, ISWC '13, Zurich, Switzerland, pp. 65-68, 2013.

[21] G. M. Antonio and B. Oswaldo, "Magnetic fields from skeletal muscles: a valuable physiological measurement?", Frontiers in Physiology, vol. 6, pp. $228(1-4), 2015$. 